SPECTROSCOPIC EVIDENCE FOR A 5.4 MINUTE ORBITAL PERIOD IN HM CANCRI

This article has been downloaded from IOPscience. Please scroll down to see the full text article.

2010 ApJ 711 L138

(http://iopscience.iop.org/2041-8205/711/2/L138)

The Table of Contents and more related content is available

Download details:

IP Address: 131.215.193.213

The article was downloaded on 26/03/2010 at 21:26

Please note that terms and conditions apply. 


\title{
SPECTROSCOPIC EVIDENCE FOR A 5.4 MINUTE ORBITAL PERIOD IN HM CANCRI
}

\author{
Gijs H. A. Roelofs ${ }^{1}$, Arne Rau ${ }^{2,3}$, Tom R. Marsh ${ }^{4}$, Danny Steeghs ${ }^{4}$, Paul J. Groot ${ }^{5}$, and Gijs Nelemans ${ }^{5}$ \\ ${ }^{1}$ Harvard-Smithsonian Center for Astrophysics, 60 Garden Street, Cambridge, MA 02138, USA; groelofs@cfa.harvard.edu \\ 2 Astronomy Department, California Institute of Technology, Pasadena, CA 91125, USA \\ ${ }^{3}$ Max-Planck Institute for Extra-Terrestrial Physics, Giessenbachstr. 1, 85748 Garching, Germany; arau@mpe.mpg.de \\ ${ }^{4}$ Department of Physics, University of Warwick, Coventry, CV4 7AL, UK \\ ${ }^{5}$ Department of Astrophysics, Radboud University Nijmegen, P.O. Box 9010, 6500 GL, Nijmegen, The Netherlands \\ Received 2009 November 30; accepted 2010 February 5; published 2010 February 23
}

\begin{abstract}
HM Cancri is a candidate ultracompact binary white dwarf with an apparent orbital period of only 5.4 minutes, as suggested by X-ray and optical light-curve modulations on that period, and by the absence of longer-period variability. In this Letter, we present Keck-I spectroscopy which shows clear modulation of the helium emission lines in both radial velocity and amplitude on the 5.4 minute period and no other. The data strongly suggest that the binary is emitting He I 4471 from the irradiated face of the cooler, less massive star, and He II 4686 from a ring around the more massive star. From their relative radial velocities, we measure a mass ratio $q=0.50 \pm 0.13$. We conclude that the observed 5.4 minute period almost certainly represents the orbital period of an interacting binary white dwarf. We thus confirm that HM Cnc is the shortest period binary star known: a unique test for stellar evolution theory, and one of the strongest known sources of gravitational waves for LISA.
\end{abstract}

Key words: binaries: close - gravitational waves - stars: individual (HM Cancri, V407 Vulpeculae) - X-rays: binaries

\section{INTRODUCTION}

Two interacting binary stars have been discovered which appear to have orbital periods shorter than 10 minutes: V407 Vul, with a period of 9.5 minutes (Motch et al. 1996; Cropper et al. 1998), and HM Cnc, with a period of 5.4 minutes (Israel et al. 2002). The uniquely short period of 5.4 minutes, if it is the orbital period, implies that HM Cnc must have formed from two white dwarfs, driven together as a result of gravitationalwave radiation. It may currently be experiencing stable mass transfer through Roche lobe overflow. Because it is potentially so extreme and unique, substantial effort has been put into unveiling HM Cnc's true nature, but as yet without conclusive results.

The key observational data show that: (1) there is no evidence for variability on periods other than 5.4 and 9.5 minutes in HM Cnc and V407 Vul, respectively (Ramsay et al. 2000, 2002a); (2) the optical flux maxima lead the X-ray maxima by about $90^{\circ}$ in both systems (Barros et al. 2007); and (3) the periods are decreasing in both systems (Strohmayer 2002, 2003, 2004, 2005; Hakala et al. 2003, 2004; Israel et al. 2004).

Three competing models have been proposed for V407 Vul and HM Cnc. One of them, the "Intermediate Polar (IP)" model, predicts that these systems are not in fact ultracompact binaries but have rather mundane orbital periods of several hours. The ultrashort periods then represent the spins of magnetic white dwarfs (Norton et al. 2004). The X-rays as well as the variable optical flux originate from the accretion flow crashing onto the magnetic poles of the magnetic white dwarf during part of the accretor's spin cycle. The spin-up of the X-ray and optical periods may be expected since the magnetic white dwarf is accreting matter of high specific angular momentum. The absence of variability on the much longer orbital period could be explained if the orbital planes of both systems are viewed exactly face-on. The observed phase offsets between the optical and X-ray light curves are difficult to explain (Norton et al. 2004), and the emission lines are unusually weak for IPs; in
V407 Vul there appear to be no emission lines at all (Ramsay et al. 2002b; Steeghs et al. 2006).

The second, "Unipolar Inductor (UI)" model, is essentially a more energetic version of the Jupiter-Io system (Wu et al. 2002; Dall'Osso et al. 2006, 2007). A magnetic white dwarf is orbited by another, non-magnetic one; the magnetic field induces an electrical potential across the non-magnetic white dwarf that sets up currents along magnetic flux tubes connecting the two stars. Ohmic dissipation of these currents in the flux tubes' footpoints on the magnetic star gives rise to the X-rays. In this model, the 5.4 minute period is the orbital period, but the two stars are detached. Since the binary loses angular momentum due to gravitational-wave radiation, it is expected to evolve toward shorter orbital periods, as observed. The main problem is the offset between the optical and X-ray flux, which requires the footpoints on the magnetic star's surface to be almost $90^{\circ}$ ahead of the orbiting non-magnetic star in both systems (Barros et al. 2005, 2007).

The third, "AM CVn" model, has a Roche lobe-filling white dwarf losing mass to a more massive white dwarf (Marsh \& Steeghs 2002; Ramsay et al. 2002b, or Cropper et al. 1998 for a magnetic version). The orbital period is 5.4 minutes in the case of HM Cnc, and the accretion stream hits the accretor directly without forming a disk. The phase offset between optical and X-ray flux is naturally accounted for, since the accretion stream will deflect and hit the accretor off-axis. The absence of longer periods is also expected, but the observed decrease of the periods in HM Cnc and V407 Vul is considered problematic for this model (Strohmayer 2002, 2004), although solutions to this problem have been put forward (Deloye \& Taam 2006; D'Antona et al. 2006; G. H. A. Roelofs \& C. J. Deloye 2010, in preparation).

This Letter describes our search for kinematic evidence, using phase-resolved spectroscopy of HM Cnc, for or against these different models. Section 2 describes our observations and data reduction. We present our results in Section 3 and discuss their implications in Section 4. 
Table 1

Log of Our Keck/LRIS Observations of HM Cnc

\begin{tabular}{|c|c|c|c|c|}
\hline Date & UT & $\begin{array}{c}\text { Spectra } \\
(60 \mathrm{~s})\end{array}$ & Weather & $\begin{array}{r}\text { Total S/N } \\
\text { (at } 4200 \AA \text { ) } \\
\end{array}$ \\
\hline $2009 / 01 / 26$ & $08: 10-12: 30$ & 136 & 1". 1 , absorption early & 78 \\
\hline $2009 / 01 / 27$ & $07: 24-13: 14$ & 223 & $1^{\prime \prime} .1$, clear & 115 \\
\hline $2009 / 03 / 31$ & $05: 52-06: 50$ & 41 & 1".3, clear & 41 \\
\hline
\end{tabular}

\section{OBSERVATIONS AND DATA REDUCTION}

After unsuccessful attempts in 2005, 2006, and 2007 to obtain phase-resolved spectroscopy of HM Cnc, we finally met acceptable weather conditions at Keck-I on 2009 January 25 and 26 and 2009 March 30. We took spectra using a 1".5 slit and the 600 grooves $\mathrm{mm}^{-1}$ grism on the blue side of the Low-Resolution Imaging Spectrograph (LRIS; Oke et al. 1995), for an effective spectral resolution of about $300 \mathrm{~km} \mathrm{~s}^{-1}$. In order to resolve the 321.5-s period in HM Cnc, we limited the exposure times to 60 s. The blue CCD, with a plate scale of 0 ' $^{\prime} 135$ pixel $^{-1}$, was binned $4 \times 4$ pixels to reduce the read-out noise while still sampling resolution elements with approximately 2 (binned) pixels. The binning furthermore reduced the dead-time between exposures to $27 \mathrm{~s}$.

Data reduction was done using standard IRAF tasks. To optimally extract the very faint individual spectra (HM Cnc has optical magnitudes $U \approx 19.6, B \approx 20.7$; Israel et al. 2002), reference aperture profiles were created from averaged blocks of typically $\sim 30$ successive frames to increase the photon statistics and remove cosmic rays from the profile. Spectra were grouped together based on the requirement that spatial center and profile (seeing) on the CCD be (near-)identical. Wavelength calibration was achieved using $\mathrm{HgCdZnNeAr}$ arc exposures. A fit of $30 \mathrm{arc}$ lines left $0.35 \AA$ root-mean-square residuals. No detectable arc drift occurred during the night, and the drift between the two nights was well below 1 pixel. We thus applied a common dispersion solution to all spectra, only applying a small shift to the first night's spectra to tie the strong [O I] 5577 sky emission lines together. The relative wavelength calibration of all spectra is estimated to be better than $0.1 \AA$.

The time stamp and velocity scale of each spectrum were transformed to the solar system's barycenter. Time stamps were checked several times during our run using Universal Time clocks available online and estimated to be correct to $1 \mathrm{~s}$ (or better). The spectrophotometric standard star Feige 34 was used for calibrating the instrumental response; variable transparency and seeing precluded an absolute flux calibration. Table 1 summarizes our observing log.

\section{RESULTS}

\subsection{Average Spectrum}

The average Keck spectrum of HM Cnc is shown in Figure 1. As previously reported (Israel et al. 2002), the spectrum is dominated by ionized helium emission lines. Their full width at half-maximum (FWHM) of $\sim 2500 \mathrm{~km} \mathrm{~s}^{-1}$ is well resolved by the $300 \mathrm{~km} \mathrm{~s}^{-1}$ resolution of our spectra. Lines of neutral helium are also present but much weaker. Our higher-quality spectrum confirms earlier findings that the even-term transitions of the He II Pickering series are stronger than the odd-term ones (see Figure 1), which was interpreted by Norton et al. (2004) and Reinsch et al. (2007) as evidence for the presence of hydrogen. In particular, the absence of He II 4200 in our spectrum is striking,

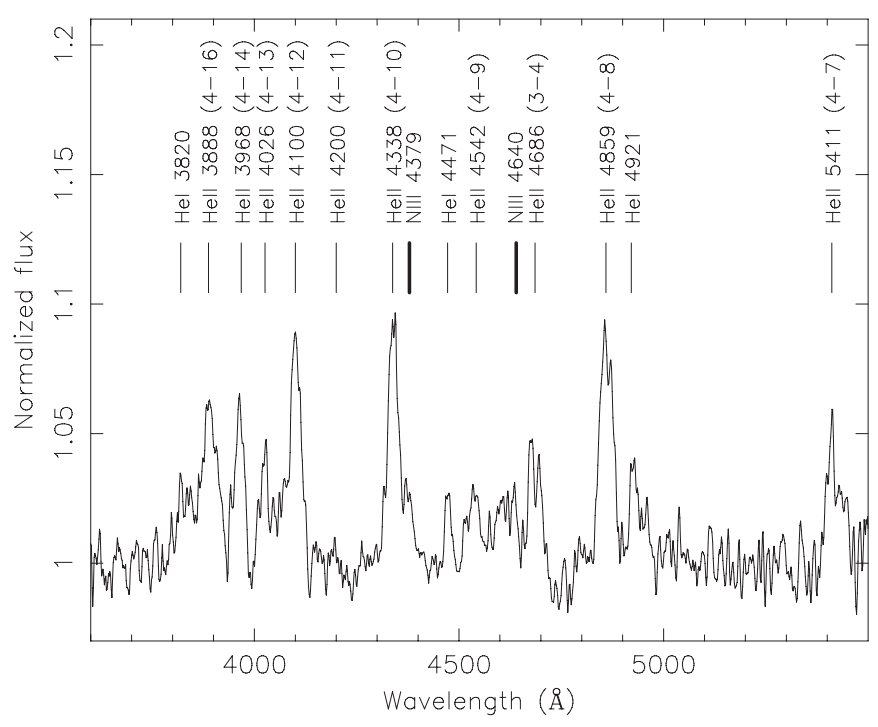

Figure 1. Average Keck-I/LRIS spectrum of HM Cnc, normalized to the continuum. The most obvious spectral lines are labeled. The He II 3888 and 4026 lines may contain a contribution from coincident He I lines.

and most easily explained if one assumes that there is indeed hydrogen in HM Cnc (for simplicity, we will refer to He II Pickering lines in the remainder of this Letter).

In addition to the $\mathrm{N}$ III 4640 line in the Bowen blend identified by Israel et al. (2002), the strong N III 4379 line appears to be present to the red of He II 4338. Apparent emission lines near $4600 \AA$ and $4800 \AA$ could be due to N IV, while a faint feature to the red of He I 4471 may be $\mathrm{N}$ III 4514 . The presence of several nitrogen lines appears to be at odds with the suggestion (based on fits to X-ray spectra) that HM Cnc may have an unusual chemical abundance pattern and be underabundant in nitrogen in particular (Strohmayer 2008). We note that nitrogen-rich matter would be expected due to the CNO-cycle if $\mathrm{HM} \mathrm{Cnc} \mathrm{is} \mathrm{an}$ ultracompact binary, unless the material experienced helium burning (Yungelson 2008), and that nitrogen indeed appears to be abundant in most AM CVn stars (see Roelofs et al. 2009).

\subsection{Ephemeris and Period Derivative}

When we phase-fold our spectra using the ephemeris of Barros et al. (2007), we observe continuum flux variations that lag slightly $\left(\sim 30^{\circ}\right.$ in phase) behind Barros et al. (2007)'s photometry. This is not unexpected given, in particular, the uncertainty in the period derivative measured by Barros et al. (2007), which translates into a phase uncertainty of about this magnitude.

By matching the phases of the variable $g$-band flux in our time-resolved spectra with the $g$-band photometry of Barros et al. (2007), we can obtain a refined ephemeris for HM Cnc. This will not be a very precise refinement since our spectra only marginally sample the orbital period, and since atmospheric transparency and seeing were variable. However, because we 

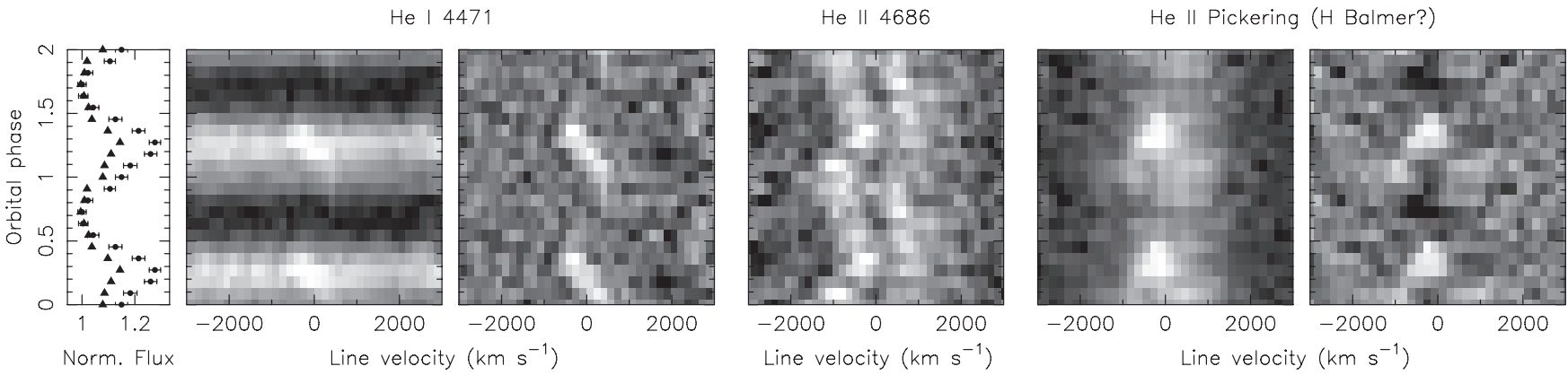

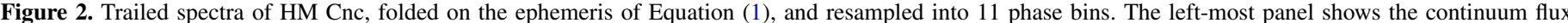

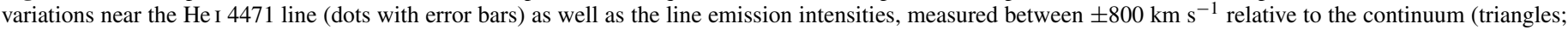

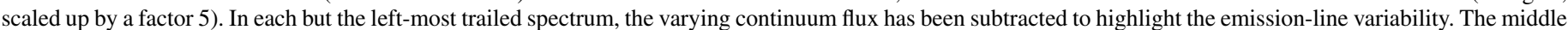

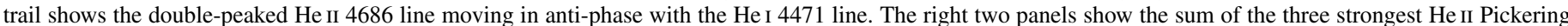

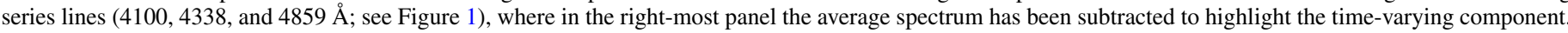
The gray-scales are linear between the dimmest and brightest pixels, where the brightest pixels have the highest flux densities

have hundreds of spectra and the folding period is much shorter than the typical timescales for sky quality variations, we can still resolve this shift. The easiest way to correct the phase shift is to reduce the frequency $v$ and its time derivative from Barros et al. (2007) by approximately $1.3 \sigma$ :

$$
\begin{aligned}
t_{0}(\text { barycentric } \mathrm{TDB}) & \equiv 53009.889943753, \\
v(\mathrm{~Hz}) & =0.00311013811(10), \\
d v / d t\left(\mathrm{~Hz} \mathrm{~s}^{-1}\right) & =3.57(2) \times 10^{-16},
\end{aligned}
$$

where numbers in parentheses are the uncertainties in the corresponding number of last decimals. The new error on the frequency derivative is estimated as the error that gives the same phase uncertainty (at our epoch) as the error on the frequency. The error on the frequency has been kept the same. This ephemeris is used throughout this Letter.

\subsection{Time-resolved Spectrum}

The left panels of Figure 2 show the time-resolved (trailed) spectrum of HM Cnc around the He I 4471 line, folded on the above ephemeris (Equation (1)). The continuum flux is seen to follow broadly the same pattern as in Barros et al. (2007) after aligning the phases: during a cycle the continuum flux increases slowly and decreases more rapidly. The scatter in our continuum fluxes, shown in the left-most panel of Figure 2, is due to differences in the average sky quality between phase bins rather than due to noise in individual spectra.

Clear spectral line variations are seen in the He I 4471 line, in the form of an "S-wave" feature that is visible for about half the period while it moves from redshifted to blueshifted wavelengths. The intensity of the S-wave in the He I 4471 line follows the intensity of the variable continuum flux, suggesting that they originate from the same region. In the remaining panels of Figure 2, the intrinsically variable continuum flux has been fitted and subtracted to highlight the line variability. Here, the He II 4686 line is plotted as well as the sum of the three strongest He II Pickering series lines (4100, 4338, and $4859 \AA$; see Figure 1). The He II lines behave quite differently from the He I 4471 line. The He II 4686 line is double-peaked and appears to wobble in anti-phase with respect to the He I 4471 line. The He II Pickering lines have a strongly modulated, narrow component which may be moving from blueshifted to redshifted in phase with (and at the same radial velocity of) the He II 4686 line. In addition, the He II Pickering lines have a fairly constant, broad component with a FWHM of $\sim 2500 \mathrm{~km} \mathrm{~s}^{-1}$, which matches the FWHM of the (double-peaked) He II 4686 profile.

We measure, from a linear back-projection Doppler tomogram (Marsh \& Horne 1988), a radial velocity semi-amplitude of $390 \pm 40 \mathrm{~km} \mathrm{~s}^{-1}$ for the S-wave feature in the He I 4471 line (corrected for a $\sim 5 \%$ bias due to finite exposure times, which we modeled using synthetic data). The error was estimated from a large ensemble of Doppler tomograms calculated from bootstrap samples of our 400 spectra. For the double-peaked He II 4686 line moving in anti-phase, we similarly measure a radial velocity semi-amplitude of $260 \pm 40 \mathrm{~km} \mathrm{~s}^{-1}$. The two peaks in the profile are redshifted and blueshifted by $650 \pm 50 \mathrm{~km} \mathrm{~s}^{-1}$.

Finally, the spectra were phase-folded on trial periods of up to $6 \mathrm{hr}$, but no radial velocity or intensity variations in the spectral lines were seen on periods other than 5.4 minutes.

\section{DISCUSSION AND CONCLUSIONS}

\subsection{The Nature of HM Cnc}

The observed radial velocity variations in the spectral lines strongly suggest that HM Cnc has a 5.4 minute orbital period. We favor the semi-detached binary white dwarf (or AM CVn) model, shown in Figure 3. It is the only model that can account for the spectroscopy presented in this Letter. The IP model predicts an orbital period of hours rather than minutes, and the observed line kinematics do not match predictions by Norton et al. (2004) for spectral line variability on the accretor's spin period (if any). We see the broad, fairly constant (and in the case of He II 4686, double-peaked) He II emission as a clear signature of accretion, which is not predicted by the UI model.

We conclude that HM Cnc is by far the shortest period binary star known. Its direct progenitor was probably a detached binary white dwarf, since evolutionary models with initially nondegenerate donor stars cannot reach such short orbital periods (e.g., Yungelson 2008; van der Sluys et al. 2005).

The observed decrease of HM Cnc's 5.4 minute period, considered the main obstacle for the AM CVn model, may be expected naturally if it is a semi-detached binary white dwarf with this orbital period, due to the relatively long mass-transfer turn-on timescales predicted for fairly low-mass helium donors (Deloye \& Taam 2006; G. H. A. Roelofs \& C. J. Deloye 2010, in preparation). 


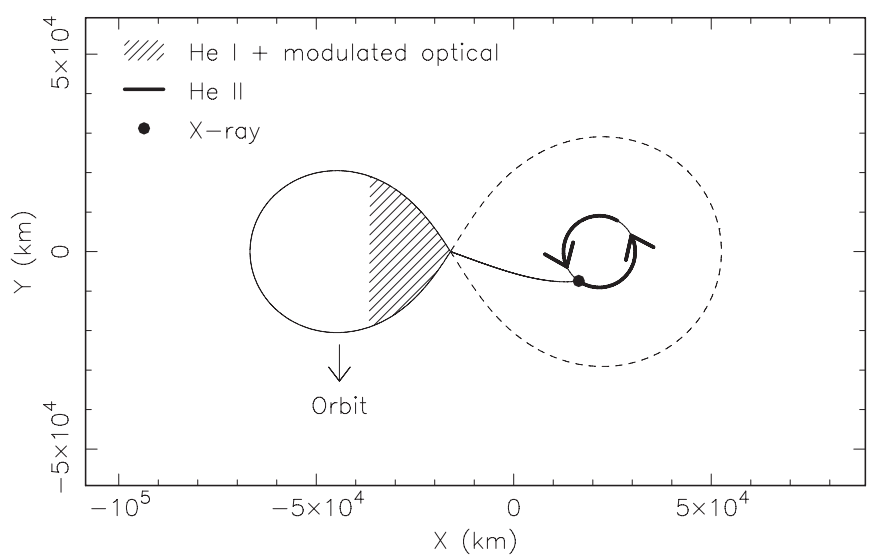

Figure 3. "AM CVn" model of HM Cnc, with the donor star on the left, transferring mass to the accretor. The impact of the accretion stream into the accretor causes the X-rays. The irradiated face of the donor star is the source of the He I 4471 emission line, and the main source of the modulated optical emission. An equatorial belt or disk around the accretor is the source of the He II emission lines. Masses $M_{2}=0.27 M_{\odot}, M_{1}=0.55 M_{\odot}$ are assumed (see the text); the center of mass is at the origin. The orbital phase (from Equation (1)) would be $\sim 0.35$ as viewed from the bottom of this page.

\subsection{System Parameters}

Based on the radial velocities of the He I 4471 and He II 4686 emission lines moving in anti-phase, we can estimate the ratio of donor to accretor mass $q \equiv M_{2} / M_{1}$ in HM Cnc. The He I 4471 line seems to originate from the irradiated face rather than the exact mass center of the donor star, and so we must estimate a "K-correction" (e.g., Muñoz-Darias et al. 2005) to obtain the donor's actual projected orbital velocity from the observed emission-line velocity. Without a $K$-correction, we get an upper limit of $q \leqslant 0.67 \pm 0.12$. Assuming that the He I line originates from the inner Lagrange point (the maximum conceivable $K$-correction) gives a lower limit $q \geqslant 0.33 \pm 0.06$. The true value will be in between; assuming a flat probability density distribution between our upper and lower limits gives $q=0.50 \pm 0.13$.

Figure 4 shows a combined Doppler tomogram of the He I 4471 and He II 4686 lines, showing the (putative) irradiated donor star and ring-like emission centered on the (proposed) accreting star. The mass ratio derived above can easily be estimated from this figure. A further interesting feature is the "bright spot" in the He II 4686 emission, occurring in the lower left quadrant of the Doppler tomogram. The velocity vectors from the center of mass to the donor star and to this He II 4686 bright spot make a $110^{\circ}$ angle approximately. Kinematically, this bright spot matches with a spot approximately on the side of the accreting star as seen from the donor, if the material in the bright spot is moving at roughly half the Keplerian (breakup) velocity near the surface of the accretor (but depending on its mass). An accretion stream impact spot at this location was proposed by Barros et al. (2007) to explain the relative phases of the optical and X-ray light curves. Assuming $M_{2}=0.13 M_{\odot}$, which is the minimum donor star mass corresponding to a Roche lobe-filling degenerate helium object, an accretor mass $M_{1} \approx 0.55 M_{\odot}$ is required to get an accretion stream impact spot sufficiently on the side of the accretor, as shown by Barros et al. (2007). Our mass ratio $q=0.50 \pm 0.13$ implies that both the donor and the accretor have to be more massive than this to still produce an impact spot sufficiently on the side (but see Wood 2009 for a possible relaxation of this constraint).

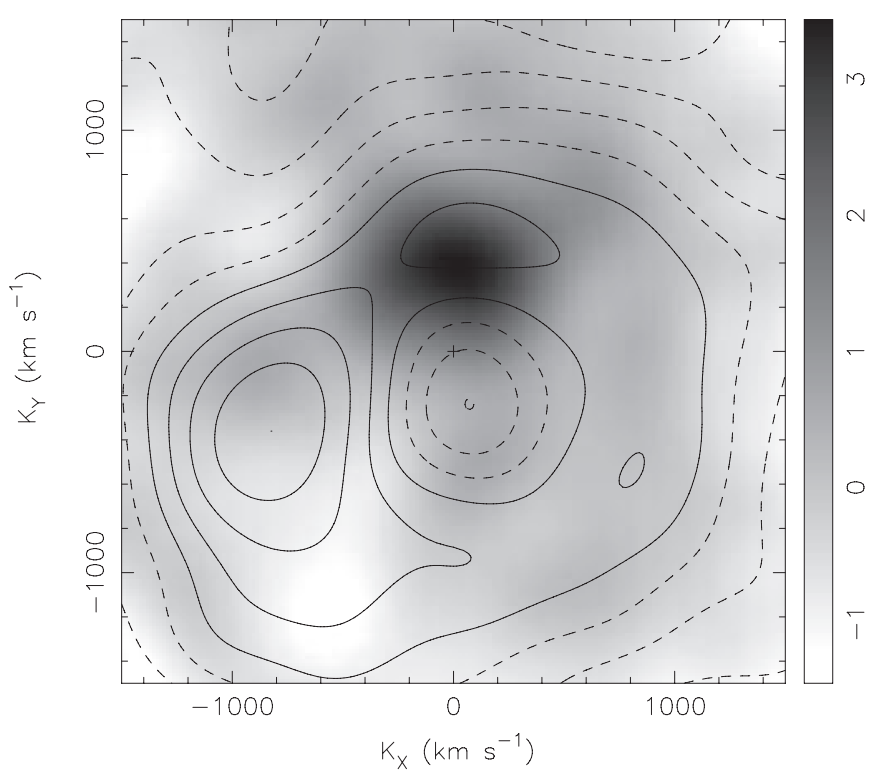

Figure 4. Doppler tomograms of He I 4471 (gray-scale) and He II 4686 (contours). The (assumed) irradiation-induced He I 4471 emission from the secondary star has been aligned with the positive $K_{Y}$-axis. The gray-scale indicates the flux overdensity as a percentage of the continuum. He II 4686 is emitted in a ring centered on the expected location of the primary star, for mass ratios $q=0.50 \pm 0.13$. Solid and dashed contours indicate where the He II 4686 flux rises above and drops below the average in the map, respectively. A bright spot occurs on the left side of the ring, approximately $110^{\circ}$ ahead of the secondary star.

The measured rate of change of the orbital period in HM Cnc (Equation (1)) also suggests that the donor star has to be significantly more massive than its fully degenerate value, if one assumes that the rate of change matches the secular rate set by gravitational-wave radiation (neglecting mass transfer). Given $q \approx 0.50$, masses $M_{2}=0.27, M_{1}=0.55$ would be required to yield the observed period derivative. We note that these are quite typical masses for binary white dwarfs according to theoretical models (Nelemans et al. 2001). Assuming instead $M_{2}=0.13 M_{\odot}$, which is again the minimum mass of a Roche lobe-filling helium donor, an accretor mass $M_{1}=1.3 M_{\odot}$ would be required. This is incompatible with our mass ratio measurement. Note that mass transfer reduces the orbital period change rate relative to the case of a detached binary, so that higher masses would be required; but in practice, it is likely that the mass transfer is not yet sufficiently developed to have a big influence (Deloye et al. 2007). We can conclude that the donor star is probably at least twice as massive as a fully degenerate helium donor would be. For masses $M_{2}=0.27 M_{\odot}, M_{1}=$ $0.55 M_{\odot}$ and assuming that the radial velocity semi-amplitude of the wobbling He II 4686 line represents the projected orbital velocity of the accretor, we obtain an inclination $i \approx 38^{\circ}$ of the orbital plane.

\subsection{HM Cnc as a Gravitational-wave Source}

With the system parameters derived in the previous section, we can calculate the gravitational-wave strain amplitude at Earth. The distance to $\mathrm{HM} \mathrm{Cnc} \mathrm{is} \mathrm{the} \mathrm{largest} \mathrm{remaining} \mathrm{un-}$ certainty; it is probably $\sim 5 \mathrm{kpc}$ based on the expected sizes and measured temperatures of the white dwarf components (Barros et al. 2007). This gives a dimensionless gravitationalwave strain amplitude $h \simeq 1.0 \times 10^{-22}$. This, together with the design sensitivities of space-borne gravitational-wave detectors 
like LISA, makes HM Cnc one of the easiest detectable sources of gravitational waves known (see Nelemans 2009).

We thank Susana Barros for helping us with the ephemeris of HM Cnc. G.H.A.R. was supported by NWO Rubicon grant 680.50.0610 to G. H. A. Roelofs. A.R. acknowledges support through NASA grant NNX08AK66G. The data presented in this Letter were obtained at the W. M. Keck Observatory, which is operated as a scientific partnership among the California Institute of Technology, the University of California, and the National Aeronautics and Space Administration. The Observatory was made possible by the generous financial support of the W. M. Keck Foundation.

\section{REFERENCES}

Barros, S. C. C., Marsh, T. R., Groot, P., Nelemans, G., Ramsay, G., Roelofs, G., Steeghs, D., \& Wilms, J. 2005, MNRAS, 357, 1306

Barros, S. C. C., et al. 2007, MNRAS, 374, 1334

Bildsten, L., Shen, K. J., Weinberg, N. N., \& Nelemans, G. 2007, ApJ, 662, L95

Cropper, M., Harrop-Allin, M. K., Mason, K. O., Mittaz, J. P. D., Potter, S. B., \& Ramsay, G. 1998, MNRAS, 293, L57

Dall'Osso, S., Israel, G. L., \& Stella, L. 2006, A\&A, 447, 785

Dall'Osso, S., Israel, G. L., \& Stella, L. 2007, A\&A, 464, 417

D’Antona, F., Ventura, P., Burderi, L., \& Teodorescu, A. 2006, ApJ, 653, 1429

Deloye, C. J., \& Taam, R. E. 2006, ApJ, 649, L99

Deloye, C. J., Taam, R. E., Winisdoerffer, C., \& Chabrier, G. 2007, MNRAS, 381,525

Hakala, P., Ramsay, G., \& Byckling, K. 2004, MNRAS, 353, 453

Hakala, P., Ramsay, G., Wu, K., Hjalmarsdotter, L., Järvinen, S., Järvinen, A. \& Cropper, M. 2003, MNRAS, 343, L10
Israel, G. L., et al. 2002, A\&A, 386, L13

Israel, G. L., et al. 2004, Mem. Soc. Astron. Ital. Suppl., 5, 148

Marsh, T. R., \& Horne, K. 1988, MNRAS, 235, 269

Marsh, T. R., \& Steeghs, D. 2002, MNRAS, 331, L7

Motch, C., Haberl, F., Guillout, P., Pakull, M., Reinsch, K., \& Krautter, J. 1996, A\&A, 307, 459

Muñoz-Darias, T., Casares, J., \& Martínez-Pais, I. G. 2005, ApJ, 635, 502

Nelemans, G. 2009, Class. Quantum Gravity, 26, 094030

Nelemans, G., Portegies Zwart, S. F., Verbunt, F., \& Yungelson, L. R 2001, A\&A, 368, 939

Norton, A. J., Haswell, C. A., \& Wynn, G. A. 2004, A\&A, 419, 1025

Oke, J. B., et al. 1995, PASP, 107, 375

Ramsay, G., Cropper, M., Wu, K., Mason, K. O., \& Hakala, P. 2000, MNRAS, 311,75

Ramsay, G., Hakala, P., \& Cropper, M. 2002a, MNRAS, 332, L7

Ramsay, G., Wu, K., Cropper, M., Schmidt, G., Sekiguchi, K., Iwamuro, F., \& Maihara, T. 2002b, MNRAS, 333, 575

Reinsch, K., Steiper, J., \& Dreizler, S. 2007, in ASP Conf. Ser. 372, Spectral Analysis and Constraints on the Nature of the Ultra-Compact Binary RX J0806.3+1527, ed. R. Napiwotzki \& M. R. Burleigh (San Francisco, CA: ASP), 419

Roelofs, G. H. A., et al. 2009, MNRAS, 394, 367

Steeghs, D., Marsh, T. R., Barros, S. C. C., Nelemans, G., Groot, P. J., Roelofs, G. H. A., Ramsay, G., \& Cropper, M. 2006, ApJ, 649, 382

Strohmayer, T. E. 2002, ApJ, 581, 577

Strohmayer, T. E. 2003, ApJ, 593, L39

Strohmayer, T. E. 2004, ApJ, 610, 416

Strohmayer, T. E. 2005, ApJ, 627, 920

Strohmayer, T. E. 2008, ApJ, 679, L109

van der Sluys, M. V., Verbunt, F., \& Pols, O. R. 2005, A\&A, 440, 973

Wood, M. A. 2009, MNRAS, 395, 378

Wu, K., Cropper, M., Ramsay, G., \& Sekiguchi, K. 2002, MNRAS, 331, 221

Yungelson, L. R. 2008, Astron. Lett., 34, 620 